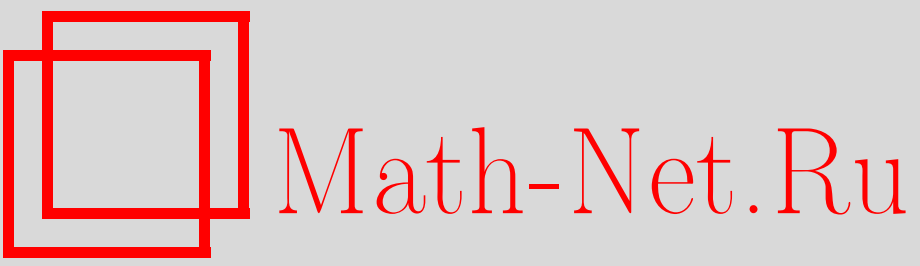

Б. К. Буздов, Об одной модели замораживания живой биологической ткани, Итоги науки и техн. Сер. Соврем. мат. и ее прил. Темат. обз., 2021, том 190, 14-18

DOI: https://doi.org/10.36535/0233-6723-2021-190-14-18

Использование Общероссийского математического портала Math-Net.Ru подразумевает, что вы прочитали и согласны с пользовательским соглашением

http://www. mathnet.ru/rus/agreement

Параметры загрузки:

IP : 34.227 .88 .159

26 апреля 2023 г., 13:15:19 


\title{
ОБ ОДНОЙ МОДЕЛИ ЗАМОРАЖИВАНИЯ ЖИВОЙ БИОЛОГИЧЕСКОЙ ТКАНИ
}

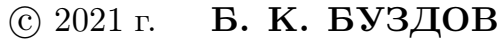

\begin{abstract}
АннотАция. В работе приводится новая математическая модель, соответствующая замораживанию живой биоткани криоаппликатором формы прямоугольного параллелепипеда, а также метод ее численного исследования, основанный на применении локально-одномерных разностных схем «сквозного счета». Модель представляет собой трехмерную трехфазную краевую задачу типа Стефана и имеет приложение в криохирургии. Специальная нелинейная зависимость источников тепла от искомого температурного поля позволяет учесть реально наблюдаемый на практике эффект пространственной локализации тепла.
\end{abstract}

Ключевые слова: математическое моделирование, криомедицина, криобиология, задача Стефана.

\section{ON ONE MODEL OF FREEZING BIOLOGICAL LIVING TISSUES}

\author{
(c) 2021 B. K. BUZDOV
}

\begin{abstract}
In this paper, we present a new mathematical model of freezing a living biological tissue by a cryoapplicator and a method for its numerical study based on the use of local one-dimensional difference schemes. The model is a three-dimensional three-phase boundary-value Stefan-type problem and has applications in cryosurgery. A special nonlinear dependence of heat sources on the unknown temperature field allows one to take into account the spatial effect of heat localization.
\end{abstract}

Keywords and phrases: mathematical modeling, cryomedicine, cryobiology, Stefan problem.

AMS Subject Classification: 97M60

1. Введение. Математическое моделирование процесса теплопроводности при охлаждении и замораживании живой биологической ткани (активной, не инертной среды) приводит к постановкам задач типа Стефана, характерной особенностью которых является существование стационарных решений. Стабилизация во времени поля температуры достигается в связи с тем, что отводимый поток тепла компенсируется возникающими в биоткани источниками тепла, обусловленными крово- и лимфотоком, метаболизмом, окислительными химическими реакциями, а также теплом, выделяемым при замерзании внеклеточной и внутриклеточной жидкости. На сегодняшний день существует большое количество научных публикаций (см., например, цитируемую литературу в [6]), в которых рассматриваются математические модели криодеструкции биологической ткани. Однако в подавляющем их большинстве за основу математической модели принимается уравнение Пеннеса [8], из которого виден линейный характер зависимости источников тепла биоткани от искомого температурного поля. Такой характер зависимости не позволяет описать реально наблюдаемую пространственную локализацию тепла. Кроме того, модель Пеннеса не учитывает того факта, что замерзание межклеточной жидкости происходит гораздо раньше, чем замерзание внутриклеточной жидкости и соответствующее этим двум процессам тепло выделяется в разные моменты времени. Приводимая ниже модель учитывает указанные особенности. 
2. Постановка задачи. Когда криоинструмент формы прямоугольного параллелепипеда «вдавливается» в биоткань (считаем, что с биотканью контактируют боковые грани параллелепипеда и верхнее основание), модель теплопроводности будет представлять собой следующую трехмерную краевую задачу типа Стефана:

$$
\begin{aligned}
& \frac{\partial}{\partial x}\left[\lambda(u) \frac{\partial u}{\partial x}\right]+\frac{\partial}{\partial y}\left[\lambda(u) \frac{\partial u}{\partial y}\right]+\frac{\partial}{\partial z}\left[\lambda(u) \frac{\partial u}{\partial z}\right]-c(u) \rho(u) \frac{\partial u}{\partial t}= \\
& =-\omega(u)+P \frac{\partial u}{\partial t} \delta\left(u-u_{1}\right)+P_{0} \frac{\partial u}{\partial t} \delta\left(u-u_{2}\right), \quad(x, y, z) \in \Omega, \quad t>0, \\
& u(x, y, z, 0)=\bar{u}=\mathrm{const}, \quad(x, y, z) \in \Omega, \\
& \lambda(u) \frac{\partial u}{\partial z}-\alpha u=-\alpha u_{A}, \quad z=c, \quad-a \leqslant x \leqslant a, \quad-b \leqslant y \leqslant b, \quad t>0, \\
& \lambda(u) \frac{\partial u}{\partial n}-\beta u=-\beta u_{A}, \quad(x, y, z) \in \Gamma_{0}, \quad t>0, \\
& \lambda(u) \frac{\partial z}{\partial n}-\gamma u=-\gamma u_{c}, \quad z=0, \quad(x, y) \in \Gamma_{1}, \quad t>0, \\
& u(x, y, z, t)=\bar{u}=\mathrm{const}, \quad(x, y, z) \in \Gamma_{2}, \quad t>0, \\
& \left.\begin{array}{l}
u\left(x, y, z_{1}(x, y, t), t\right)=u_{1} \\
u\left(x, y, z_{2}(x, y, t), t\right)=u_{2}
\end{array}\right\}
\end{aligned}
$$

Здесь $\Omega=\Omega_{1} \backslash \Omega_{0}$, где $\Omega_{1}=\{(x, y, z) \in[-A, A] \times[-B, B] \times[0, C]\}, \Omega_{0}=\{(x, y, z) \in[-a, a] \times[-b, b] \times$ $[0, c]\} . A, B, C, a, b, c$ известные положительные постоянные, причем $a<A, b<B, c<C$; $\Gamma_{0}$ боковая поверхность параллелепипеда $\Omega_{0} ; \Gamma_{1}$ нижнее основание параллелепипеда $\Omega_{1}$ без нижнего основания параллелепипеда $\Omega_{0} ; \Gamma_{2}$ граница области $\Omega_{1}$ без нижнего основания; $\bar{n}$ внутренняя нормаль области $\Omega ; \lambda(u), c(u), \rho(u)$ коэффициенты теплопроводности, теплоемкости и плотности биоткани соответственно; $u_{A}=u_{A}(t)$ температура охлаждающей поверхности криоинструмента; $u_{c}$ температура окружающей среды; коэффициент теплообмена $\alpha$ является постоянной величиной; коэффициент теплообмена $\beta=\beta(z)$ монотонно возрастающая функция переменной $z$ (вид ее должен определяться экспериментально); $\gamma$ коэффициент теплообмена с окружающей средой; $\bar{u}$ температура биоткани, до которой еще не дошел холод; $u_{1}$ температура замерзания межклеточной жидкости, $u_{2}$ температура замерзания внутриклеточной жидкости (температура криопоражения биоткани); $\delta(x)$ дельта-функция Дирака.

Определению подлежит функция температуры $u=u(x, y, z, t)$, а также пара изотермических поверхностей $z_{1}(x, y, z), z_{2}(x, y, z)$, на которых температура биоткани равна, соответственно, $u_{1}$, $u_{2} ; A, B, C$ постоянные, характеризующиеся тем, что вне рассматриваемого параллелепипеда температура биоткани постоянна и равна $\bar{u}$.

Предполагается, что коэффициенты $c(u), \rho(u), \lambda(u)$ могут иметь разрывы типа скачка при $u=u_{1}$ и $u=u_{2}$, а также, что

$$
c(u) \geqslant c_{\min }>0, \quad \rho(u) \geqslant \rho_{\min }>0, \quad \lambda(u) \geqslant \lambda_{\min }>0
$$

После ввода функции теплосодержания $(\eta(x)$ функция Хевисайда)

$$
H(u)=\int_{0}^{u} c(\xi) \rho(\xi) d \xi+P \eta\left(u-u_{1}\right)+P_{0} \eta\left(u-u_{2}\right)
$$

уравнение (1) примет вид

$$
\frac{\partial}{\partial x}\left[\lambda(u) \frac{\partial u}{\partial x}\right]+\frac{\partial}{\partial y}\left[\lambda(u) \frac{\partial u}{\partial y}\right]+\frac{\partial}{\partial z}\left[\lambda(u) \frac{\partial u}{\partial z}\right]-\frac{\partial H(u)}{\partial t}=-\omega(u) .
$$

Добавив к уравнению (2) начальные и граничные условия и сглаживая разрывные функции [3], задачу можно решать численно, применяя локально- одномерные разностные схемы [7]. 
3. Источники тепла. Как было отмечено выше, характерной особенностью процесса теплопроводности при охлаждении живой биоткани является стабилизация к предельному стационарному состоянию с четкой пространственной локализацией температурного поля. Указанная особенность моделируется специальным образом подобранной нелинейной функциональной зависимостью источников тепла от температуры. По теплофизическому смыслу функциональная зависимость источников тепла от температуры должна быть ограниченной, непрерывной и монотонно убывающей в интервале положительных температур, а в интервале отрицательных температур ограниченной и монотонно возрастающей [1]. Также необходимым является условие вертикального вхождения кривой $\omega=\omega(u)$ в точку $(\bar{u}, 0)$, т.е. необходимо, чтобы $\omega^{\prime}(\bar{u})=-\infty$. Оно имеет следующий смысл при сколь угодно малом возмущении начальной температуры биоткани в ней мгновенно возникают источники тепла.

При проведении численных расчетов на ЭВМ можно использовать следующие функции источников:

$$
\begin{aligned}
& \omega_{1}(u)= \begin{cases}\omega_{0}(\bar{u}-u)^{\beta}, & u_{1} \leqslant u<\bar{u}, \\
0, & \text { иначе }\end{cases} \\
& \omega_{2}(u)= \begin{cases}w_{0} \ln (\bar{u}-u), & u_{1} \leqslant u<\bar{u} \\
0, & \text { иначе }\end{cases} \\
& \omega_{3}(u)= \begin{cases}w_{0}(\bar{u}-u)^{\beta}, & u_{1} \leqslant u<\bar{u} \\
-\frac{w_{0} \bar{u}^{\beta}}{u_{A}}\left(u-u_{A}\right), & u_{A} \leqslant u<u_{1} . \\
0, & u<u_{A}\end{cases}
\end{aligned}
$$

Содержащиеся в указанных зависимостях параметры $\omega_{0}$ и $0<\beta<1$ должны определяться экспериментально.

4. Разностная схема. Введем сетку:

$$
\begin{aligned}
W_{h}=\left\{\left(x_{i}, y_{j}, z_{k}\right), i\right. & i=1, \ldots, N_{1}, j=1, \ldots, N_{2}, k=1, \ldots, N_{3}, x_{0}=-A, y_{0}=-B, \\
& \left.z_{0}=0, x_{N_{1}}=A, y_{N_{2}}=B, z_{N_{3}}=C, x_{i}=x_{0}+i h, y_{j}=y_{0}+j h, z_{k}=k h\right\} .
\end{aligned}
$$

При этом будем считать, что отрезки $[-a, a],[-b, b],[0, c]$ разбиты, соответственно, на $m_{1}, m_{2}, m_{3}$ частей; $m_{1}<N_{1}, m_{2}<N_{2}, m_{3}<N_{3}$. Разбиение проведем так, чтобы величины

$$
\frac{N_{i}+m_{i}}{2}, \quad i=1,2,3
$$

были целыми. Очевидно, это всегда возможно.

Обозначим $\nu_{i j k}^{n}$ значение искомой функции в точке $\left(x_{i}, y_{j}, z_{k}\right)$ в момент времени $t_{n}=n \tau$. Пусть $\nu_{i j k}^{n+1 / 3}, \nu_{i j k}^{n+2 / 3}$ значения искомой функции на промежуточных слоях. Локально-одномерная разностная схема будет иметь следующий вид:

$$
\begin{gathered}
\frac{1}{3} B\left(\nu_{i j k}^{n+1 / 3}\right) \cdot \frac{\nu_{i j k}^{n+1 / 3}-\nu_{i j k}^{n}}{\tau}=\Lambda_{1} \nu_{i j k}^{n+1 / 3}+\frac{1}{3} \omega\left(\nu_{i j k}^{n+1 / 3}\right), \quad\left(x_{i}, y_{j}, z_{k}\right) \in W_{h}, \\
\nu_{i j k}^{n+1 / 3}=\bar{u}, \quad i=0, \quad i=N_{1}, \quad 0<j<N_{2}, \quad 0<k<N_{3}, \\
\Lambda_{1} \nu_{i j k}^{n+1 / 3}=\frac{1}{h}\left(\lambda\left(\nu_{i+1 / 2, j k}^{n+1 / 3}\right) \frac{\nu_{i+1, j, k}^{n+1 / 3}-\nu_{i j k}^{n+1 / 3}}{h}-\lambda\left(\nu_{i-1 / 2, j k}^{n+1 / 3}\right) \frac{\nu_{i j k}^{n+1 / 3}-\nu_{i-1, j k}^{n+1 / 3}}{h}\right), \\
\frac{1}{3} B\left(\nu_{i j k}^{n+2 / 3}\right) \cdot \frac{\nu_{i j k}^{n+2 / 3}-\nu_{i j k}^{n+1 / 3}}{\tau}=\Lambda_{2} \nu_{i j k}^{n+2 / 3}+\frac{1}{3} \omega\left(\nu_{i j k}^{n+2 / 3}\right), \quad\left(x_{i}, y_{j}, z_{k}\right) \in W_{h}, \\
\Lambda_{2} \nu_{i j k}^{n+2 / 3}=\frac{1}{h}\left(\lambda\left(\nu_{i, j+1 / 2, k}^{n+2 / 3}\right) \frac{\nu_{i+1, j, k}^{n+2 / 3}-\nu_{i j k}^{n+2 / 3}}{h}-\lambda\left(\nu_{i, j-1 / 2, k}^{n+2 / 3}\right) \frac{\nu_{i j k}^{n+2 / 3}-\nu_{i-1, j k}^{n+2 / 3}}{h}\right),
\end{gathered}
$$




$$
\begin{aligned}
& \nu_{i j k}^{n+2 / 3}=\bar{u}, \quad j=0, \quad j=N_{2}, \quad 0 \leqslant i \leqslant N_{1}, \quad 0 \leqslant k \leqslant N_{3}, \\
& \frac{1}{3} B\left(\nu_{i j k}^{n+1}\right) \cdot \frac{\nu_{i j k}^{n+1}-\nu_{i j k}^{n+2 / 3}}{\tau}=\Lambda_{3} \nu_{i j k}^{n+1}+\frac{1}{3} \omega\left(\nu_{i j k}^{n+1}\right), \quad\left(x_{i}, y_{j}, z_{k}\right) \in W_{h}, \\
& \Lambda_{3} \nu_{i j k}^{n+1}=\frac{1}{h}\left(\lambda\left(\nu_{i j, k+1 / 2}^{n+1}\right) \frac{\nu_{i+1, j, k}^{n+1}-\nu_{i j k}^{n+1}}{h}-\lambda\left(\nu_{i j, k-1 / 2}^{n+1}\right) \frac{\nu_{i j k}^{n+1}-\nu_{i-1, j k}^{n+1}}{h}\right) \text {, } \\
& \nu_{i j 0}^{n+1}=\frac{\lambda\left(\nu_{i j 0}^{n+1}\right)}{\gamma h+\lambda\left(\nu_{i j 0}^{n+1}\right)} \cdot \nu_{i j 1}^{n+1}+\frac{\gamma h u_{c}}{\gamma h+\lambda\left(\nu_{i j 0}^{n+1}\right)}, \quad 0<i<\frac{N_{1}-m_{1}}{2}, \quad \frac{N_{1}+m_{1}}{2}<i<N_{1} \text {, } \\
& 0<j<\frac{N_{2}-m_{2}}{2}, \quad \frac{N_{2}+m_{2}}{2}<j<N_{2}, \\
& \nu_{\frac{N_{1}+m_{1}}{2}, j, k}^{n+1 / 3}=\frac{\lambda\left(\nu_{\frac{N_{1}+m_{1}}{2}, j, k}^{n+1 / 3}\right)}{\alpha h+\lambda\left(\nu_{\frac{N_{1}+m_{1}}{2}, j, k}^{n+1 / 3}\right)} \cdot \nu_{\frac{N_{1}+m_{1}}{2}+1, j, k}^{n+1 / 3}+\frac{\alpha h u_{A}}{\alpha h+\lambda\left(\nu_{\frac{N_{1}+m_{1}}{2}, j, k}^{n+1 / 3}\right)}, \\
& \frac{N_{2}-m_{2}}{2}<j \leqslant \frac{N_{2}+m_{2}}{2}, \quad 0<k<m_{3}, \\
& \nu_{\frac{N_{1}-m_{1}}{2}, j, k}^{n+1 / 3}=\frac{\lambda\left(\begin{array}{c}
\nu_{\frac{N_{1}-m_{1}}{2}, j, k}^{n+1 / 3} \\
2
\end{array}\right)}{\lambda\left(\nu_{\frac{N_{1}-m_{1}}{2}, j, k}^{n+1 / 3}\right)-\alpha h} \cdot \nu_{\frac{N_{1}-m_{1}}{2}-1, j, k}^{n+1 / 3}-\frac{\alpha h u_{A}}{\lambda\left(\nu_{\frac{N_{1}-m_{1}}{2}, j, k}^{n+1 / 3}\right)-\alpha h}, \\
& \frac{N_{2}-m_{2}}{2}<j \leqslant \frac{N_{2}+m_{2}}{2}, \quad 0<k<m_{3}, \\
& \nu_{i, \frac{N_{2}+m_{2}}{2}, k}^{n+2 / 3}=\frac{\lambda\left(\nu_{i, \frac{N_{2}+m_{2}}{2}, k}^{n+2 / 3}\right)}{\alpha h+\lambda\left(\nu_{i, \frac{N_{2}+m_{2}}{2}, k}^{n+2 / 3}\right)} \cdot \nu_{i, \frac{N_{2}+m_{2}}{2}+1, k}^{n+2 / 3}+\frac{\alpha h u_{A}}{\alpha h+\lambda\left(\nu_{i, \frac{N_{2}+m_{2}, k}{2}}^{n+2 / 3}\right)}, \\
& \frac{N_{1}-m_{1}}{2}<i<\frac{N_{1}+m_{1}}{2}, \quad 0<k<m_{3},
\end{aligned}
$$

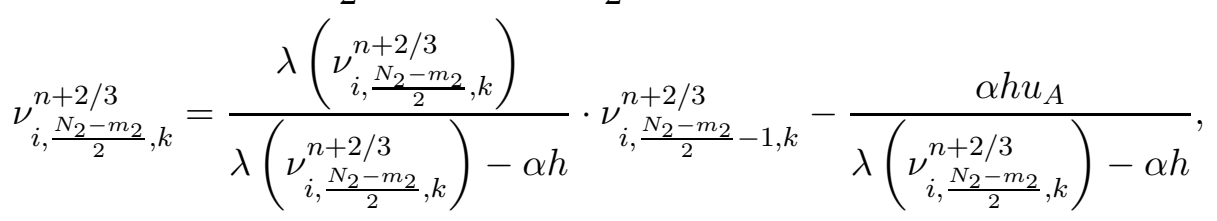

$$
\begin{aligned}
& \frac{N_{1}-m_{1}}{2}<i<\frac{N_{1}+m_{1}}{2}, \quad 0<k<m_{3}, \\
& \nu_{i, j, m_{3}}^{n+1}=\frac{\lambda\left(\nu_{i, j, m_{3}}^{n+1}\right)}{\alpha h+\lambda\left(\nu_{i, j, m_{3}}^{n+1}\right)} \cdot \nu_{i, j, m_{3}+1}^{n+1}+\frac{\alpha h u_{A}}{\alpha h+\lambda\left(\nu_{i, j, m_{3}}^{n+1}\right)}, \quad \frac{N_{1}-m_{1}}{2}<i<\frac{N_{1}+m_{1}}{2}, \\
& \frac{N_{2}-m_{2}}{2}<j<\frac{N_{2}+m_{2}}{2}, \\
& \nu_{i j N_{3}}^{n+1}=\bar{u}, \quad 0 \leqslant i \leqslant N_{1}, \quad 0 \leqslant j \leqslant N_{2}, \\
& \nu_{i j k}^{0}=\bar{u}, \quad 0 \leqslant i \leqslant N_{1}, \quad 0 \leqslant j \leqslant N_{2}, \quad 0 \leqslant k \leqslant N_{3} \text {. }
\end{aligned}
$$

Здесь

$$
\begin{aligned}
& \nu_{i+1 / 2, j, k}=0,5\left(\nu_{i j k}+\nu_{i+1, j k}\right), \\
& \nu_{i, j+1 / 2, k}=0,5\left(\nu_{i j k}+\nu_{i, j+1, k}\right), \\
& \nu_{i, j, k+1 / 2}=0,5\left(\nu_{i j k}+\nu_{i j, k+1}\right),
\end{aligned}
$$




$$
B(u)=H^{\prime}(u) .
$$

Из написанных выше уравнений видно, что для нахождения значения сеточной функции $\nu$ на $(n+$ 1)-м временном слое по известному значению на $n$-м временном слое необходимо последовательно решать три серии одномерных задач, соответственно, по координатам $x, y, z$. Каждая такая задача представляет собой нелинейную алгебраическую систему с трехдиагональной матрицей, и для ее решения лучше пользоваться методом прогонки совместно с каким-либо итерационным методом, например, методом Ньютона. При определении $\nu^{n+1}$ коэффициенты $c, \rho, \lambda$ можно брать на предыдущей итерации.

5. Заключение. В заключение отметим ряд особенностей задач типа Стефана, возникающих в криомедицине.

1. Одной из важных особенностей является реально наблюдаемый эффект пространственной локализации тепла, связанный с наличием в биоткани нелинейных источников тепла специального вида. Этот эффект, хотя и усложняет математическую модель (в уравнении появляется нелинейный член, зависящий от искомого температурного поля), позволяет перейти от постановок задач в неограниченной области к постановкам в ограниченной области.

2. Нелинейные источники тепла зависят от числовых параметров, которые должны определяться экспериментально, что само по себе представляет самостоятельную достаточно сложную задачу.

3. Использующийся для решения метод сквозного счета с предварительным сглаживанием разрывных функций не зависит от размерности задачи.

4. Связь между шагами по времени и пространству, обнаруженная при решении ряда одномерных и двумерных задач $[4,5]$, указывает на то, что построенные разностные схемы являются условно-устойчивыми.

5. Стабилизация, как поля температуры, так и поля изотерм, к предельному стационарному состоянию наступает примерно через 6 мин. (значения теплофизических характеристик используемых при проведении численных экспериментов можно взять в [2]).

\section{СПИСОК ЛИТЕРАТУРЫ}

1. Березовский $A$. А. Одномерная локальная задача Стефана плоскопараллельной криодеструкции биологической ткани// в кн.: Задачи теплопроводности с подвижными границами. - Киев: Ин-т мат. АН УССР, 1985. - С. 38.

2. Березовский А. А., Леонтъев Ю. В. Математическое прогнозирование криовоздействия на биологические ткани// в кн.: Криобиология. - Киев: Наукова думка, 1989. - 3. - С. 713.

3. Будак Б. М., Соловъева E. Н., Успенский А. Б. Разностный метод со сглаживанием коэффициентов для решения задачи Стефана// Ж. вычисл. мат. мат. физ. - 1965. - 5. - С. 828840.

4. Буздов Б. К. Моделирование криодеструкции биологической ткани// Мат. модел. - 2011. - 23, № 3. - C. 2737.

5. Буздов Б. К. Численное исследование одной двумерной математической модели с переменным коэффициентом теплообмена, возникающей в криохирургии // Сиб. ж. индустр. мат. -2017 . -20 , № 4 . C. 2228.

6. Жмакин А. И. Физические основы криобиологии// Усп. физ. наук. -2008 . - 178, № 3. - С. 243266.

7. Самарский $A$. А. Локально-одномерные разностные схемы на неравномерных сетках// Ж. вычисл. мат. мат. физ. - 1963. - 3, № 3. - С. 431466.

8. Pennes H. H. Analysis of tissue and arterial blood temperature in the resting human forearm// J. Appl. Physiol. - 1948. - 1. - P. 93102.

Буздов Беслан Каральбиевич

Институт информатики и проблем регионального управления,

Кабардино-Балкарский научный центр РАН, Нальчик

E-mail: beslan.buzdov@yandex.ru 